\title{
Artículo
}

\section{La triple combinación de roles: mujeres-madres-estudiantes universitarias}

\section{Aportes para (re)conocer la noción política de sus trayectorias}

The triple combination of roles: women-mothers- college students

Contributions to recognize the political notion of their trajectories

Lucía Pilar Cosciuc

Licenciada en Trabajo Social

(Universidad Nacional de Rosario)

Investigadora Centro de Investigaciones

Feministas y Estudios de Género (CIFEG)

(Universidad Nacional de Rosario)

Correo: luciacosciuc@gmail.com 
La “triple combinación de roles": mujeres-madres-estudiantes universitarias.

Aportes para (re)conocer y recuperar sus vivencias.

Lucía Cosciuc

\title{
Resumen
}

El presente artículo es un resumen de mi tesina de Grado de la Licenciatura en Trabajo Social, titulada "Eran insostenibles todos los roles. Reflexiones y aportes para (re)conocer las vivencias de mujeres-madres-estudiantes universitarias" (2018). Versa sobre la relación entre maternidad y formación universitaria. El objetivo es reconocer la noción política de las trayectorias de vida de mujeres que transitan, al mismo tiempo, su carrera universitaria y su proyecto de maternidad, entendiendo que las tensiones y dificultades que se les presentan al asumir dicha 'triple combinación de roles' se desprenden de las propias estructuras opresivas del sistema patriarcal que jerarquizan diferencias y generan, a partir de ello, desigualdades en función, principalmente, del género y la clase, entre otros aspectos.

\section{Palabras clave}

Maternidad, Estudiantes universitarias, Trayectorias de vida, Roles sociales.

\begin{abstract}
This article is a review of my bachelor's degree thesis on Social Work titled "All the roles were unsustainable" (2018). It is about the relationship between motherhood and collage education. The objective is to recognize the political notion of the life trajectories of women who are simultaneously pursuing their university career and their motherhood project, understanding that the tensions and difficulties they face when assuming this 'triple combination of roles' arise from the oppressive structures of the patriarchal system that hierarchize differences and generate, from this, inequalities based mainly on gender and class, among other aspects.
\end{abstract}

\section{Keywords}

Motherhood, Female university students, Life trajectories, Social roles. 


\section{Introducción. En primera persona}

El recorrido por la Universidad siempre es un camino incierto, plagado de sentidos, de historias y vivencias, que dejan un aprendizaje en cada paso: en todos los aspectos en los que la palabra aprendizaje puede entenderse. Cuando este recorrido se transita siendo mujer y asumiendo no sólo el rol de estudiante, sino que a su vez se "habita" el rol de madre el camino a trazar tendrá implicaciones que si bien serán diferentes para las vivencias de cada una de estas mujeres, tendrá como denominador común una vivencia que puede preverse cargada de dificultades y obstáculos.

Mi propio transitar por la Universidad Nacional de Rosario (Argentina), en la carrera de Trabajo Social y el hecho de haber tenido a mi hija durante ese recorrido, me llevó a vivenciar en la piel esta experiencia, este recorrido particularizado, personalizado. Y, con ello, a elaborar, en cada paso y valiéndome de diferentes redes de apoyo, estrategias o herramientas que me permitieran cumplir con las exigencias de ambos roles.

Los imperativos sociales y las estructuras culturales naturalizadas juegan un papel determinante en los recorridos de las mujeres que somos además madres y estudiantes: nos exigen ciertas pautas, tareas y plazos propios de cada uno de esos roles para poder considerarnos "exitosas" en ambos.

A los fines del presente artículo, que se trata como bien se anticipó de un resumen de tesina de grado de la Licenciatura en Trabajo Social, titulada "Eran insostenibles todos los roles. Reflexiones y aportes para (re)conocer las vivencias de mujeres-madres-estudiantes universitarias" $(2018)^{1}$, se buscará dar cuenta de cómo operan esas estructuras patriarcales que subordinan, excluyen y oprimen a las mujeres y que, por tanto, dificultan y obstaculizan las trayectorias de vida de aquellas que asumen, además, los roles de madres y estudiantes universitarias. Para ello, lo central será reconocer que es en los relatos de experiencias y trayectorias de vida de estas mujeres en donde se encuentran los insumos teóricos que deben ser recuperados por

1 Disponible en: $\underline{\text { https://1library.co/document/z3dlixdy-insostenibles-reflexiones-aportes-conocer-vi- }}$ vencias-mujeres-estudiantes-universitarias.html 
su valor político y exponencial para pensar-elaborar, en un futuro inmediato, nuevas políticas educativas.

En este sentido, los límites de extensión del presente artículo circunscriben el recorrido teórico y metodológico al objetivo de introducir aspectos de la problemática citada que argumenten y visibilicen mecanismos sistemáticos de exclusión para la vida de las mujeres, particularmente en el entrecruzamiento de sus proyectos de estudios universitarios y sus maternidades.

$\mathrm{Al}$ pensar en estructuras que determinan las trayectorias de vida de estudiantes-madres, fue imprescindible reconocer(me) en principio en el cumplimiento de estos roles: en las expectativas, las exigencias, las restricciones, las prescripciones que asumí, que asumo, y que tantas/os otros/as entendieron que yo misma debía asumir. Desde ese lugar, pude despegarme de aquellas premisas impuestas, limitantes y estrictas, para comenzar a visibilizar mis propios andares mucho más complejos, más sentidos y re-significados, y especialmente, internamente habitados y vivenciados en primera persona.

\section{El sentido académico}

Interpelar, deconstruir y desmenuzar los roles sociales que asumimos es el primer ejercicio a realizar para poder comenzar a entender cómo los mismos funcionan y cómo son validados y reproducidos por el propio sistema social-político-patriarcal que nos contiene. Entender que ninguna categoría es ingenua y que, en consecuencia, la lectura de las imposiciones sociales y comportamientos esperados para cualquier tipo de rol debe asumirse de una forma crítica, política y cuestionadora es el siguiente paso afirmativo en este sentido.

Poder pensar(me) y pensar a otras mujeres habitando en sus cuerpos estos roles es justamente el inicio de la problematización que permite comenzar a entender cómo las exigencias en torno a ellos operan. Reflexionar acerca de cómo son las trayectorias de vida y cómo construimos nuestras subjetividades dentro y fuera de los roles es lo que me permite poder ir más allá en el reconocimiento de actrices/actores, intereses e injerencias que se ponen en juego en un espacio específicamente habitado, en esta lectura, por mujeres-madres-estudiantes. 
En este aspecto, y sin temor a dudas, considero que para poder hablar de las trayectorias de vida de estas mujeres no existe relato más "autorizado" y fidedigno que el de ellas mismas; el de recuperar la voz de esas vivencias, de esas historias de vidas que resignificarán todo lo que pueda verse en primera instancia como un sentido atribuido (y por ende "no propio"). Si bien dichas voces no serán recuperadas en este estudio, es desde allí que considero necesario recuperar su aporte en términos político-teóricos y problematizar el modo en el que los roles se habitan en el cuerpo; cómo se reconstruyen y revalorizan en cada vivencia y cómo se apropia ese sentido en las historias construidas.

Entender entonces, tal como desarrolla Sandra Harding (1993), que es la experiencia de las mujeres la base o el sustento que debe utilizarse para el desarrollo de investigaciones (especialmente feministas) partiendo del privilegio epistemológico de tales experiencias como conocimiento situado social e históricamente. Es decir, que las propias experiencias de mujeres, en donde se contemplan entrecruzamientos de género, clase y raza, generan aportes mucho más reveladores, críticos e influyentes para las agendas de investigación que las producidas de forma parcial o limitada por varones; quienes justamente sitúan sus experiencias en un lugar de dominación en relación a las mujeres.

\section{Nivel del estudio y aspectos metodológicos}

Debido a que la temática que planteo en este estudio no presenta antecedentes análogos en Argentina (de acuerdo a la recuperación bibliográfica llevada a cabo al momento de elaborarlo), es que el mismo se desarrolla en un nivel exploratorio, con la pretensión de constituirse como antecedente para abordar dicha temática en estudios posteriores en donde se llegue a profundizar el nivel de investigación, se consulten nuevas fuentes y perspectivas de análisis, abordaje, etc.

En este sentido, el presente artículo busca sembrar algunas consideraciones generales respecto a las tensiones, dificultades y obstáculos que se les presentan a las mujeres-madres-estudiantes universitarias en función del género y de otros entrecruzamientos de dominación y opresión al asumir 
dicha combinación de roles sociales. La mencionada intención se lleva a cabo entonces a través de una revisión y recuperación bibliográfica, a modo de conceptualizar desde diferentes autores/as y propuestas teóricas algunas categorías claves para pensar tales trayectorias de vida desde una perspectiva de género que exponga no sólo cómo operan las exigencias y tareas asociadas a los géneros sino también cómo se gestan a partir de allí las condiciones de opresión, invisibilización y marginación a las que son sometidas las mujeres.

Además de dicha revisión, se ofrecen fundamentos conceptuales que permiten reconocer, en términos de producción de conocimiento, los aportes de las experiencias y trayectorias de vida de las mujeres-madres-estudiantes universitarias en su valor político, como fuentes privilegiadas, por su condición de tales, en la mencionada producción.

Este ordenamiento teórico y reflexivo podría entonces preparar el terreno para estudios futuros sobre esta temática en particular que contribuyan a la elaboración de políticas educativas universitarias inclusivas que tomen como insumo el aporte proveniente de la experiencia de las propias estudiantes-madres.

\section{Pensar (con) perspectiva de género y (en)clave feminista}

La utilización de un abordaje teórico con perspectiva de género es transversal a todo el recorrido del presente estudio y para ello cito, en primer término, la propuesta de Donna Haraway, quien sostiene que el

(...) género es un concepto desarrollado para contestar la naturalización de la diferencia sexual en múltiples terrenos de lucha (...) [tratando] de explicar y de cambiar los sistemas históricos de diferencia sexual, en los que los hombres y las mujeres están constituidos y situados socialmente en relaciones de jerarquía y antagonismo (1995:221).

Desde este lugar, visualizo cómo el concepto de género permite corrernos de esa naturalización de roles y expectativas impuestas para hombres y mujeres, y comenzar a pensar nuestra sociedad en términos y categorías históricas, y por lo tanto cambiantes. Esta primera aproximación permite dar 
cuenta entonces que, por su carácter histórico, las condiciones de posibilidad de las experiencias de mujeres, particularmente madres y estudiantes, en un sistema que las oprime, margina e invisibiliza, pueden, y deben, ser transformadas para que ya no sea reproducido, en esta lógica, como una estructura de dominación de unos sobre otras.

Comprender que en términos de género las elaboraciones culturales no son estables o permanentes podría ser el primer paso que se puede dar a la hora de visibilizar, en particular, las vivencias de las mujeres como sujetas de opresión a lo largo de la historia y en todos los ámbitos y espacios posibles. Desde aquí es que es posible significar la subordinación femenina como una estructura funcional al sistema hegemónico patriarcal que aún con "modificaciones", permanece vigente.

La perspectiva de género invita a reconocer la distinción y diferenciación sexual (y binaria) entre hombres y mujeres como un

(...) fenómeno de carácter cultural (lo que se concibe como "masculino" y "femenino" no es natural ni universal sino que es construido y difiere según las culturas, las razas, las religiones), histórico (lo que cada cultura entiende como "masculino" y "femenino" varía de acuerdo a los diferentes momentos históricos) y relacional (lo que se entiende por "masculino" se define en relación a lo que se entiende por "femenino", y viceversa, en un horizonte de significaciones mutuas (Fabbri, 2014:145).

Es posible, gracias a este abordaje, quitarle el sesgo universal, atemporal o natural a toda tarea, estereotipo o rol social asociados y asignados a varones/mujeres por su condición de tales. Lo que se pretende aquí es visibilizar la conformación de estas construcciones vinculadas al género de las personas como productos del propio sistema patriarcal que, como tal, producen marginalidad y opresión en particular para las trayectorias de vida de las mujeres. Por otro lado, se busca exponer la importancia de abandonar la lógica que pretende aún hoy hacernos creer que existe una única forma, preestablecida y prescriptiva, de ser mujeres y varones. 


\section{Referentes teóricos}

Al hablar de la recuperación de trayectorias de vida, entendidas como “(...) relatos de vida de sujetos que las reconstruyen discursivamente, y exponen formas de actuar, sentidos, valores, formas de concebir la realidad" (Lera et al, 2007:33), me posiciono entendiendo esta noción conceptual como una herramienta válida y, si se quiere pertinente, a la hora de buscar una mayor comprensión de las situaciones singulares que involucran a las personas con las que trabajamos, y de cómo éstas las enfrentan o resignifican.

Pensar en cómo las mujeres que son madres y estudiantes buscan compatibilizar estos roles, debe partir necesariamente por recuperar sus trayectorias. Esta reconstrucción de andares particulares busca dar cuenta de cómo se asumen los roles sociales, considerados por Paul Horton y Chester Hunt (1986) como comportamientos o conductas que se esperan de una persona en determinado contexto, lo que a su vez, según ellos, implica saber implementar las exigencias que son socialmente construidas y saber exigir los privilegios que se ganan por el buen cumplimiento de los mismos; además de aprender las actitudes, sentimientos y expectativas que estos roles también exigen.

Las exigencias que implican asumir uno o más roles sociales, dejan entrever mecanismos que, al mismo tiempo, generan ciertos 'comportamientos esperados' por parte de quienes los asumen; por lo tanto, no cumplir con dichos comportamientos o expectativas sugeridas sería visto, en esta lógica, como una falta o falencia. ¿Para quién o ante quién(es) es esta falta? En primera instancia, para las personas a quienes se les adjudican las responsabilidades y exigencias que se establecen como propias de cada rol asumido; ante los cuales se rinde cuentas a la sociedad, que juzga la falta y sanciona en consecuencia.

Entiendo que dichos roles, como tales, son construcciones sociales y que, por ello, responden a un determinado modelo de sociedad inscripto en un determinado contexto. Por eso, analizo las referidas construcciones siempre partiendo de pensarlas como operantes en el contexto actual, situadas, y que responden a cierto ordenamiento social que (aún) hoy nos rige: el 
sistema patriarcal. ¿Qué se entiende por ello? Según Marta Fontenla (2008) el patriarcado puede definirse como un

(...) sistema de relaciones sociales sexo-políticas basadas en diferentes instituciones públicas y privadas y en la solidaridad inter-clases e intra-género instauradas por los varones, quienes como grupo social y en forma individual y colectiva, oprimen a las mujeres también en forma individual y colectiva y se apropian de su fuerza productiva y reproductiva, de sus cuerpos y sus productos, ya sea con medios pacíficos o mediante el uso de la violencia (2008:3).

En este sentido, se visualiza cómo en el interior de este sistema de dominación predominante tanto en lo cultural como en lo legal, se inscribe, en palabras de Juan Marco Vaggione, la heteronormatividad como un "conglomerado de principios morales, religiosos y legales [que] también ha ejercido influencia en el razonamiento judicial y ha fortalecido la heterosexualidad como el principio regulador en las relaciones de familia" (2008:21).

Inscriptas en esta lógica, se desprenden las exigencias en torno a los distintos roles asumidos por las mujeres, que parten de la organización del tiempo y las tareas domésticas e incluyen el cuidado y atención integral de los/as hijos/as en todas sus necesidades (salud, educación, esparcimiento, alimentación, etcétera) y/o de otro/a familiar a cargo. Incluso en los casos en los que las mujeres trabajan formalmente o se encuentran estudiando en el nivel superior, dichas exigencias las ubican como únicas o principales responsables para su cumplimiento. Por lo tanto, en primera instancia es menester reflexionar acerca de cuáles son los comportamientos socialmente esperados de una mujer que asume el rol de madre, quien dentro de una concepción patriarcal de familia debe asumir la responsabilidad principal en la esfera doméstica: cuidado de los/las hijos/as y organización del hogar.

A ello se vincula la noción de "instinto maternal" que Lorena Saletti Cuesta (2008) recupera, a través de diferentes autoras, e intenta desnaturalizar; ya que es a partir de dicha noción que la ideología patriarcal sitúa a las mujeres dentro del ámbito de la reproducción biológica, negando o desconociendo con ello su identidad por fuera de la función materna. La autora 
entiende que, construyendo la maternidad de las mujeres como natural e inevitable, se dictamina que toda mujer debe querer y deber ser madre; y con ello se consolida su localización en la esfera doméstica. La naturalización de este rol, como socialmente esperado, configura una imagen idealizada de las madres; por ende, todos aquellos comportamientos que no se incluyan dentro de esa imagen o que desvíen la atención de las mujeres sobre el cuidado de los/las hijos/as y de la organización del hogar serán concebidos como incorrectos y por fuera de lo éticamente deseable, construyendo así la contra-imagen de "malas madres" o "madres descuidadas".

Es posible ver cómo aún persisten, desde diversas perspectivas, nociones vinculadas a una prescripción y exigencia social de género que iguala la imagen de mujer $=$ madre y vincula tal significación a la plena realización de las mujeres como tales. Esta imagen de la maternidad, que deposita el deseo casi natural de toda mujer a ser madre y con ello ubica prácticamente la totalidad de las responsabilidades vinculadas a este rol sobre las mujeres; es la que todavía hoy habla de la existencia de un instinto y amor maternal como "hecho instintivo, irracional, que se supone se manifiesta desde la infancia de toda mujer" (Saletti Cuesta, 2008:170).

Vinculado a ello es que abordo la conceptualización sobre la maternidad entendiéndola, primeramente, como una construcción social y "un proceso complejo y pleno de ambigüedades que determina y configura la subjetividad de las mujeres, sean madres o no" (Palomar Verea, 2009:4). La maternidad históricamente construida permite de-construir el sinfín de imaginarios vinculados a ella a lo largo del tiempo, invita a elaborar una idea de división igualitaria y justa de responsabilidades y cuidados de los y las hijos e hijas y propone configurar el lugar de las mujeres como poseedoras de deseos y metas que pueden no vincularse de manera prioritaria a su (posible y no excluyente) rol maternal.

Entender que "el trabajo materno es y puede ser llevado a cabo por un hombre o por una mujer, desligando así el trabajo materno del sexo de quien lo lleva a cabo (...) es una práctica que, como otra cualquiera, está condicionada a un contexto social particular" (Saletti Cuesta, 2008:182); y, por lo tanto, quita en primera instancia la sobrecarga que generan todas las 
responsabilidades de cuidado sobre las mujeres. Por otro lado, esta perspectiva permite reconocer a dicho trabajo materno como una práctica que siempre debe analizarse en su contexto particular y, por tanto, no puede ser universalizada ni naturalizada en ninguna de sus formas ni tampoco asignada arbitrariamente a ninguna persona en función de su género.

Este recorrido, que permite pensar la relación entre los géneros en términos políticos por tratarse de una relación de poder y dominación, lleva además a recuperar la propuesta de la economía feminista en contraposición a la "economía convencional" en términos concretos de trabajo y empleo circunscriptos también sobre la base de esa relación entre géneros. Esto es entender, tal como reconoce Silvia Federici (2013), que con la instauración de división sexual del trabajo, por la cual los hombres se especializaron en trabajos "productivos" y remunerados en el espacio público y las mujeres en actividades de reproducción diaria y generacional de la vida en el espacio doméstico-privado; se consolidó, y legitimó, un sistema patriarcal en el que la apropiación violenta por parte de los varones del trabajo de las mujeres se volvió la fuerza productiva dominante y hegemónica y también su propio motor.

Es a partir del reconocimiento de la división sexual del trabajo desde donde estudios de la economía feminista sostienen que las sociedades industriales se han construido sobre el trabajo cotidiano no reconocido de las mujeres; es decir, el trabajo reproductivo que garantiza la sostenibilidad de la vida y que socialmente se les ha adjudicado por razón de género. Esta perspectiva da cuenta de la interrelación y dependencia productiva y reproductiva del trabajo llevado a cabo históricamente por las mujeres; lo cual sustenta además la crítica feminista a la economía convencional, para la cual no hay otro trabajo a considerar más que el asalariado, es decir aquel que se compra y vende como mercancía.

Con la consolidación entonces de las sociedades industriales, la pérdida de la tierra y los medios de existencia fueron parcialmente compensadas en un pacto interclases gestado entre varones gracias a la apropiación posterior de las mujeres, sus cuerpos y su fuerza de trabajo con la reproducción cotidiana de la vida. En este marco, el ordenamiento de la reproducción 
biológica y sexual generó además un factor clave en la vida de las mujeres que, como reconoce Federici, se trató de proceso de desposesión por otro: la invisibilización y devaluación de toda actividad reproductiva, diluida y controlada por el salario.

De acuerdo a todo este último recorrido, recupero la noción de economía del cuidado para poder dar cuenta de cómo se estructuran las tareas de cuidado y organización de hogar, y cómo desde esta lógica, dichas tareas se reservan casi de forma exclusiva a las mujeres. Esta noción contribuye entonces, tal como sostiene Valeria Esquivel, a “(...) instalar al 'cuidado' como un problema de política pública, sacándolo del terreno de lo privado y (si se insiste lo suficiente) desnaturalizándolo como lo propio de las mujeres y de los hogares" (2011:11). Esquivel afirma que se trata de un concepto mucho más potente que los utilizados con anterioridad (trabajo doméstico, no remunerado, etcétera) ya que “(...) el 'cuidado’ -más allá de si se remunera o no, más allá de si se realiza en los hogares o fuera de él- remite con más claridad a una relación interpersonal: los cuidados se brindan y se reciben" (Esquivel, 2011:10).

El concepto de economía del cuidado refiere en un sentido amplio a

(...) todas las actividades y prácticas necesarias para la supervivencia cotidiana de las personas en la sociedad en que viven. Incluye el auto-cuidado, el cuidado directo de otras personas (la actividad interpersonal de cuidado), la provisión de las precondiciones en que se realiza el cuidado (la limpieza de la casa, la compra y preparación de alimentos) y la gestión del cuidado (coordinación de horarios, traslados a centros educativos y a otras instituciones, supervisión del trabajo de cuidadoras remuneradas, entre otros). El cuidado permite atender las necesidades de las personas dependientes, por su edad o por sus condiciones/capacidades (niños y niñas, personas mayores, enfermas o con algunas discapacidades) y también de las que podrían auto-proveerse dicho cuidado (Rodríguez Enríquez, 2015:36).

Vislumbrar estos conceptos y consideraciones desde una perspectiva de género, abre la posibilidad de comprender y analizar las trayectorias de vida de las mujeres-estudiantes-madres, sus configuraciones de roles y familia, su vínculo con la maternidad y sus expectativas y deseos, desprendiéndolos de 
todo prejuicio o construcción simbólica dada o preestablecida que lleve a una lectura alejada de la propia autopercepción de estas mujeres, y del reconocimiento de sus experiencias como verdaderas construcciones de saber.

Retomando la propuesta inicial de Harding, es aquí donde se puede entrever la importancia de producir conocimiento socialmente situado y que algunas de estas localizaciones sociales, como es el caso de las trayectorias de vida de mujeres-madres-estudiantes universitarias se tornan imprescindibles e innegables como puntos de inicio para dicha construcción de conocimiento; aunque en ciertos aspectos no se ajusten a la totalidad de los requisitos estrictos del mundo científico y el pensamiento occidental. Así, la autoriza establece una lógica de descubrimiento que tiende a producir conocimiento para quienes son marginalizadas, y no para el uso exclusivo de dichos aportes por parte de grupos dominantes en sus proyectos de manejar las vidas de esas personas.

\section{Habitar la universidad: ¿qué sabemos de las y los estudiantes?}

Para poder caracterizar cómo son las y los estudiantes de educación superior, entiendo que inicialmente es importante pensar cómo las y los vemos. Es decir, poder reconocer, tal como sostiene Carlota Guzmán Gómez:

$\mathrm{Al}$ estudiante como un sujeto con necesidades particulares y con necesidades de atención (...) [reconocer] la importancia de escuchar a los estudiantes ya sea para reconocer sus problemas personales, familiares o aquellos relacionados con su trayectoria educativa, con los obstáculos a los que se han enfrentado (2011:93).

Al reconstruir el rol de estudiantes universitarias, se reflexiona acerca de cuál es la trayectoria estudiantil universitaria que se instituye como hegemónica en las universidades contemporáneas, influenciadas y resignificadas a partir de las nuevas exigencias que planea el sistema capitalista global actual. Como sostiene Manuel Escobar (2007), los programas universitarios, en gran medida, dejan ver la pretensión de subjetividades estudiantiles que serán especialmente productivas en contextos de adaptabilidad y flexibilidad laboral, construyendo una profesionalización que segmente y especialice 
conocimientos. Por otro lado, reconoce el autor, la configuración del espacio-tiempo propuesta por la universidad es regulada por ritmos claramente diferentes a los particulares de cada estudiante.

Desde esta óptica, una trayectoria estudiantil universitaria, plasmada en los planes de estudio y/o programas académicos por asignaturas, establece una cronología de trabajo que prevé la duración de la carrera y organiza ciertos requisitos curriculares que deben cumplimentarse en un tiempo determinado. El no cumplimiento de dichos requisitos o temporalidades esperadas representa entonces una discontinuidad en relación a las exigencias que implican las trayectorias estudiantiles universitarias establecidas previamente por la estructura institucional universitaria. ¿Qué significa esto? En otras palabras: una vinculación directa con la frustración académica. Nuevamente: ¿Frustración-discontinuidad-incumplimiento desde la óptica de quién(es)?

Reconocer entonces que existen estudiantes, tanto hombres como mujeres, que se convierten en los o las primeros/as de su familia en alcanzar el nivel superior de estudios; o pensar en estudiantes que deben trabajar formalmente durante el cursado de sus carreras para poder sostener sus estudios, y hasta considerar que existen estudiantes que comienzan a estudiar superada la mediana edad con hijos/as de diversas edades a cargo; invita a entender que no existe una forma única de construir la mencionada trayectoria estudiantil universitaria, sino que para abordar las particularidades de cada uno/a de ellos/as, es importante partir de pensar en un campo amplio, diverso y por supuesto, complejo de entrecruzamientos de aspectos de clase, género, origen étnico, etcétera.

Carlota Guzmán Gómez reconoce que la trayectoria educativa de estudiantes universitarios/as y los procesos de construcción de la identidad se configuran entendiendo que los/las estudiantes tienen distintas formas de vivir su condición de tales y viven múltiples y diversas experiencias. Junto con ello, entiende que es importante comprender que, a su vez, lo que ocurre en el espacio universitario es parte de la formación de los y las estudiantes, y que es allí en donde se despliegan prácticas que dan sentido a su quehacer cotidiano en este ámbito (Guzmán Gómez, 2011). La autora sostiene, finalmente, que la relación con los/as pares, los apoyos recibidos y las experien- 
cias propias de cada trayectoria estudiantil juegan en este contexto un papel importante en la permanencia de los y las estudiantes en sus carreras y en el rumbo que toman las estas trayectorias.

Se entiende entonces que existen diversas formas de transitar las trayectorias estudiantiles universitarias, y con ello, distintas e infinitas formas de habitar la universidad: ya sea a partir de las propias construcciones y significaciones internas, de las condiciones familiares y económicas y de los vínculos interpersonales celebrados entre los diversos actores que confluyen en el ámbito universitario. A partir de estas múltiples configuraciones es que se establece entonces cada trayectoria como forma particularizada de habitar el rol universitario; y en particular, pensado en el presente artículo desde la trayectoria de vida de quienes asumen la triple combinación de roles.

Asumiendo la mencionada diversidad de trayectorias estudiantiles universitarias, es que tampoco es posible, ni deseado, anticipar ni universalizar las experiencias, como bien se mencionó, en una trayectoria estudiantil única y esperada; siendo que la multiplicidad de entrecruzamientos y aspectos que se consideran al abordar la asunción del rol de estudiantes universitarios/as definen y delimitan dichas trayectorias.

En líneas generales, las carreras del nivel superior establecen una limitación en el tiempo de duración determinada en años; dentro de los cuales se deberían finalizar los estudios. Sin embargo, pocos/as son los/as estudiantes que logran (o desean) cumplir con este parámetro temporal por múltiples y diversas razones, tal como fue desarrollado.

$\mathrm{Al}$ exponer que no existe una trayectoria estudiantil universitaria única, se debe afirmar que aunque al asumir el rol de estudiante universitario/a la misma difiera de aquellas expectativas o exigencias sociales que del propio rol social se desprenden, no significa que tal trayectoria estudiantil se asocie al fracaso o a la frustración académica; sino que por el contrario debe visualizarse como un recorrido particularizado y repleto de significaciones que lo han determinado, producto, a su vez, de los múltiples aspectos que estructuran la asignación de roles sociales.

Otro aspecto fundamental (vinculado al anterior) a tener en cuenta al recuperar las trayectorias estudiantiles universitarias, y particularmente en 
La “triple combinación de roles": mujeres-madres-estudiantes universitarias.

Aportes para (re)conocer y recuperar sus vivencias.

Lucía Cosciuc

lo que concierne a este trabajo a las trayectorias de mujeres-estudiantes-madres, es el factor del tiempo y la conciliación del mismo frente a las exigencias que implica la triple combinación de roles. Existe por un lado una lógica institucional desde la cual se programa y organiza la vida académica entendida como "el tiempo estatuido" (que incluye tiempos de cursado, plazos administrativos, horarios, etcétera); y por otro, se encuentran las diferentes formas en las que

El sujeto vive el tiempo, esto es, el significado que le confiere y que le asigna en el contexto de su propia vida cotidiana y de su biografía. Este significado personal conferido por el estudiante al tiempo se expresa (...) en lo que desde el punto de vista institucional se vislumbra como una trayectoria discontinua (Mata, 2009 citado en Guzmán Gómez 2011:98).

Aquí es donde es posible percibir la distancia que existe entre la lógica institucional, rígida y preestablecida, y los diferentes significados y necesidades que presentan los y las estudiantes en torno a la organización de su tiempo.

El tiempo como tal es un elemento central en las trayectorias estudiantiles universitarias, y lo es aún más en las vidas de las mujeres-estudiantes-madres, donde la distancia entre sus necesidades y la enunciada lógica institucional se alarga. Los tiempos se desmenuzan y se fragmentan en muchas más piezas que las que se llegan a considerar desde las instituciones universitarias. $\mathrm{Y}$ esto es notable al percibir cómo estas mujeres vivencian dicha distancia, en donde los plazos no les alcanzan, los horarios se les restringen y la sobrecarga de responsabilidades, las obliga a desplegar estrategias multidimensionales para lograr acortar la brecha temporal que las aleja de una trayectoria estudiantil continua y esperada. Todo ello las significa, y hace de sus trayectorias de vida una experiencia impregnada por la configuración que cada una elabore en primera persona a partir de asumir cada uno de sus tres roles, ignorada muchas veces por las mencionadas instituciones.

Para estas estudiantes la utilización productiva del tiempo es un dispositivo que establece un salto de calidad en sus recorridos académicos. Los malabares que les permiten cumplir con las exigencias que se les presentan 
en sus hogares y en las aulas de la universidad son fundamentalmente recursos de aprovechamiento del tiempo; es decir de esos escasos instantes entre una actividad y otra, en las largas jornadas nocturnas o en la disposición de realizar multi-tareas de forma simultánea. Hablo de considerar dobles o triples jornadas de trabajo doméstico, académico y en muchos casos también laboral; en donde aun teniendo en cuenta las particularidades, el tiempo se constituye siempre como un elemento clave que determinará la vida de estas mujeres.

Este punto, que considera al tiempo como vector que define en parte las trayectorias de vida de las mujeres que aquí se mencionan, muestra cómo, una vez más, el sistema patriarcal pone en funcionamiento mecanismos que oprimen y limitan las vidas de las mujeres al invisibilizar que la disposición y organización del tiempo para ellas se reduce y limita en función de las tareas mencionadas dentro de la economía del cuidado que les son asignadas por su condición de género.

Es aquí donde surge interrogar acerca de: ¿Cómo se acorta dicha brecha de tiempo? ¿Qué rol cumplen las instituciones universitarias para contribuir a ello? Al afirmar, en primera instancia, que las trayectorias estudiantiles universitarias de mujeres madres han sido poco estudiadas y que por tal motivo desde las instituciones no se poseen herramientas o políticas claras que atañen a esta población en particular, es que sostengo que para que estas puedan contribuir a achicar la mencionada brecha, primero deben conocer cómo viven y construyen sus trayectorias las mencionadas estudiantes. Partir de allí podrá permitir que las instituciones universitarias cuenten con información desde las voces y experiencias de las estudiantes, para poder elaborar políticas específicas acordes a sus demandas y necesidades, y no al revés.

Sostiene Carlota Guzmán Gómez que, para ello, es fundamental incursionar en las prácticas cotidianas de los y las estudiantes, y también en los procesos de construcción de sentido que se dan en los contextos institucionales particularmente universitarios.

Pensar desde la diversidad de trayectorias estudiantiles universitarias y desde la particularidad de las experiencias de estudiantes-madres podría considerarse el principal eje para trabajar por la equidad educativa, lo que impli- 
ca no sólo una equidad formal sino también la incorporación de prácticas y políticas concretas que garanticen la permanencia de las estudiantes en las universidades y que se acoplen a sus necesidades. Todo ello volviendo a reconocer que las trayectorias estudiantiles universitarias de varones, por su condición de género, difieren significativamente por este y otros entrecruzamientos ya enunciados que deben ser tenidos en cuenta de las trayectorias de mujeres, en particular madres.

Se torna indispensable, en este sentido, reconocer la particularidad de las mujeres como estudiantes universitarias siendo su irrupción masiva en la educación superior un fenómeno atravesado por largas luchas de las mujeres. Esto lleva a visualizar cómo ha sido esa inclusión de las mujeres, cuáles han sido los obstáculos sorteados para lograrlo y de qué forma, aún hoy, su rol como estudiantes debe ser revalidado o defendido muchas veces en la vida y trayectoria universitaria en general, y en algunos ámbitos académicos en particular.

La construcción de sentidos al interior de cada trayectoria estudiantil universitaria recaba aspectos de las experiencias cotidianas (en este trabajo en particular de quienes asumen la triple combinación de roles); los cuales aportan significaciones valiosas que representan la configuración de su vida cotidiana, es decir en el ámbito en donde "hacen su propia historia, pero en condiciones previamente dadas" (Heller, 2004 citado en Veroneze, 2015:139).

Para hablar de la(s) subjetividad(es), Cristina Palomar Verea (2009) retoma a Rosi Braidotti (2000), al afirmar que se trata de un proceso intensivo, múltiple y discontinuo de 'llegar a ser'. De esta forma, Palomar Verea visualiza dicho proceso como un devenir o una cualidad del ser que no se tiene por el sólo hecho de nacer. Por otro lado, la autora recupera la propuesta de Alain Touraine (2007), quien reconoce que la construcción de la(s) subjetividad(es) es un reto mayor para las mujeres, debido a que éstas han tenido y tienen que desprenderse de ciertos determinantes y complejos de género que se han tejido en los imaginarios sociales: principalmente la noción de maternidad, la cual no sólo se inscribe como una práctica sobrecargada de significaciones, sino que además se plasma en el registro del cuerpo de las 
mujeres, lo que complejiza aún más la reflexión en torno a la subjetivación de las mismas.

Este último aporte conceptual en términos de construcción de subjetividades, se incorpora como herramienta de análisis para pensar y des-andar las trayectorias estudiantiles universitarias de mujeres-madres también en clave de género, continuando con la propuesta teórica-conceptual inicial que aquí se incluye. Por otro lado, permite entender que en tales procesos se ponen en juego los mecanismos, harto sabidos, que configuran las relaciones de dominación, poder y opresión que se establecen entre los géneros. El proceso de construcción de subjetividades, entonces, no hace más que evidenciar cómo las estructuras opresivas del sistema patriarcal generan y jerarquizan diferencias en razón de género-clase, las cuales luego determinan y permean las formas en las que, en particular, las mujeres-madres-estudiantes universitarias asumen sus roles y tensionan los propios obstáculos y dificultades que se les presentan al intentar compatibilizarlos.

\section{Mujeres en la universidad: breve recorrido histórico}

A la hora de hablar de la inclusión de las mujeres en las universidades, tanto a nivel global como específicamente en nuestro país, debe pensarse primeramente que el origen de la universidad reside en "un proyecto de modernidad (...) [donde] tanto el papel social de la universidad como el ejercicio de la docencia y la investigación estuvieron soportados en un sentido de pensamiento y de valores considerados universales" (Escobar, 2007:49). Además, se la ubicaba en un lugar considerado de privilegio para la conformación de un proyecto de civilización percibido como universal. Se establecía además como requisito un determinado perfil de estudiante: varón ilustrado y racional.

Dicho esto, se entiende que las universidades no eran un lugar en el que las mujeres iban a ser incluidas, por lo que la incorporación de las mismas se dio a través de un proceso sistemático que la autora Alicia Itatí Palermo (2006) definió en el texto "El acceso de las mujeres a la educación universitaria" como lento pero ininterrumpido, en el que en un primer momento se dio en forma excepcional, de algunas mujeres que ingresaban haciéndose 
La “triple combinación de roles": mujeres-madres-estudiantes universitarias.

Aportes para (re)conocer y recuperar sus vivencias.

Lucía Cosciuc

pasar por varones; y en un segundo momento, de forma más masiva, en un contexto de luchas y reclamos feministas por la igualdad de derechos ya no sólo para algunas mujeres pertenecientes a las aristocracias. Tiene su origen concreto en Estados Unidos donde las mujeres, tras la guerra civil, comenzaron a realizar tareas y actividades que hasta el momento eran ejecutadas de forma exclusiva por hombres. Luego, se intensificó el proceso en países de Europa, llegando a América Latina recién a finales del siglo XIX.

La circunscripción de las mujeres a la esfera doméstica, justificada por la estructura patriarcal, fue el primer y principal obstáculo que éstas debieron sortear; siendo que ello las privó de formar parte de la vida pública y limitó su mundo al ámbito privado y familiar vinculado a tareas y actividades "propias de su sexo". Este planteamiento, reconoce Edda Samudio (2016), fue esbozado desde diferentes autores, filósofos y pensadores reconocidos globalmente como clásicos, los cuales definieron desde diversas disciplinas cuál era el lugar que las mujeres debían ocupar en la historia, permitiendo el sostenimiento de la exclusión y marginación sistemática de las mujeres de los espacios de formación y construcción de conocimiento que, con algunos matices y modificaciones, logró perdurar en el tiempo y a lo largo y ancho de los distintos países y culturas.

\section{Alcances y futuros estudios}

El desarrollo del presente artículo pretendió, como recorte puntual del trabajo realizado en mi Tesina de grado, esbozar un marco teórico-conceptual que por un lado, contribuya a la visibilización de las múltiples dimensiones que determinan, producto de la propia estructura de dominación patriarcal, los obstáculos y dificultades que se ponen en tensión en las trayectorias de vida de las mujeres-madres-estudiantes universitarias; y, por otro lado, destacar la importancia de recuperar las experiencias de tales trayectorias (que sí fueron recabados en la Tesina) como insumos imprescindibles para la elaboración de investigaciones en torno a la temática planteada.

En este sentido, considero importante plantear cuáles podrían ser los aspectos que no han sido alcanzados por este estudio y que bien podrían ser parte de investigaciones futuras; incluso algunos ya abordados en mi Tesina. 
En tal caso, el hecho de que mi investigación haya estado centrada en experiencias de mujeres madres y estudiantes permite crear el interrogante acerca de cómo son estas trayectorias de mujeres-estudiantes-madres en las distintas facultades o institutos universitarios de nuestro país, lo que permita entonces la elaboración de líneas de investigación, propias de cada casa de estudio, que aborden y recuperen la importancia política de tales trayectorias. Con ello, se podrían desarrollar estudios comparativos, que narren las propias experiencias desde sus protagonistas y sus dificultades y necesidades, con el fin de no sólo de limitarlas a la problematización teórica, sino también elaborar políticas universitarias en este sentido.

Sería interesante, además, conocer y analizar las experiencias de paternidades de estudiantes universitarios, y establecer una investigación comparativa respecto a las trayectorias de mujeres-estudiantes-madres.

Finalmente, al reconocer la importancia de establecer líneas de investigación propias de cada facultad e instituto universitario sobre las poblaciones estudiantiles, podría ser útil conocer cuáles son las universidades que ya cuentan con herramientas o estrategias específicas de inclusión para mujeres-estudiantes-madres (tal como analicé en la Tesina sobre la Facultad de Ciencia Política y RRII de la UNR) y, a partir de allí, pensar en la reformulación o elaboración de nuevas estrategias que contribuyan a conformar instituciones universitarias inclusivas en este sentido.

Consideraciones abiertas para (re)conocer los aportes de las trayectorias de vida de mujeres-estudiantes-madres

Para dar cuenta de cómo son las trayectorias de vida de mujeres-estudiantes-madres, se sostiene que debe hacerse recuperando la noción política de dichas trayectorias desde las experiencias de sus protagonistas, fundamentalmente entendiendo que es desde allí -como se planteó- que deben pensarse y elaborarse las políticas educativas universitarias.

En este sentido, recupero la noción de Carlota Guzmán Gómez, quien afirma que para poder establecer políticas o programas que se adecúen a las necesidades de los y las estudiantes del nivel superior, es necesario en primer lugar que las instituciones educativas "construyan sus propias líneas 
La "triple combinación de roles": mujeres-madres-estudiantes universitarias.

Aportes para (re)conocer y recuperar sus vivencias.

Lucía Cosciuc

de investigación sobre sus estudiantes, lo cual implica generar fuentes de información confiables" (2011:99). Además, la autora reconoce que este tipo de líneas de investigación contribuirá no sólo a acceder a un conocimiento más completo y realista sobre las vivencias de los y las estudiantes, sino que además permitirá pasar de los supuestos a las certezas en materia de información.

Sostiene la autora que el gran reto para las instituciones universitarias a la hora de generar líneas de investigación sobre estudiantes se plasma en "lograr conocer e interpretar las necesidades propias de los estudiantes y no sólo las que resultan relevantes en términos del cumplimiento de las metas institucionales" (Guzmán Gómez, 2011:99).

Pensar y elaborar políticas, programas o estrategias en el ámbito educativo universitario, como en cualquier otro espacio en el que las mismas se gesten, debe partir de (re)conocer a quienes forman parte de dicho ámbito, en este caso, a las estudiantes universitarias que son madres, y no al revés.

\section{Reflexionando (ando)}

La implacable lucha de las mujeres por formar parte del mundo universitario, que se inició muchas décadas atrás, hoy se encuentra firme en gran parte de las universidades de nuestro país. Si bien se ha avanzado en este sentido, es menester seguir realizando aportes teóricos para construir políticas universitarias inclusivas, que recuperen las experiencias de las mujeres-madres como insumo para dicha elaboración. Como bien se abordó en el presente trabajo, urge en este sentido que se inauguren diversas líneas de investigación que aborden y recuperen estos aportes desde y con las propias estudiantes.

Hoy por hoy, se vivencia una réplica de la lucha de esas mujeres en otros ámbitos, tal vez más amplios y abarcativos. Es por eso que los feminismos buscan deconstruir todas las elaboraciones dogmáticas y teóricas del sistema patriarcal hegemónico que aún pretenden ubicar a las mujeres como principales responsables de las tareas de cuidado de los/as hijos/as y de la organización doméstica. Para las mujeres que asumen la triple combinación de roles, tales construcciones asociadas a estructuras de dominación patriarcal 
oprimen, marginan e invisibilizan sus trayectorias de vida; obstaculizando además la propia compatibilización de roles de los cuales se desprenden, propios de la estructura, expectativas e imposiciones vinculadas al género, la maternidad, la clase, etcétera.

Ante todo, son(somos) mujeres. Es otro el peso de las responsabilidades, son otros los caminos y fronteras. Son distintas y múltiples las miradas. Hay conflictos feminizados y feminizantes que indefectiblemente se deben desentramar y hacer visibles. Por este motivo, entiendo que si bien el abordaje en clave de género es necesario como primer paso para reconocer las propias opresiones y marginaciones a las que somos sometidas las mujeres y para visibilizar así las dificultades y obstáculos que se tensionan al intentar compatibilizar los roles que asumimos; cabe aclarar que las propias respuestas que habilitan a esbozar estas propuestas teóricas y epistemológicas que aportan los feminismos, deben darse al interior del Estado como responsable de la elaboración de políticas educativas concretas que garanticen la inclusión y permanencia de las estudiantes en la educación superior. Este es entonces el desafío más importante a asumir por parte de las instituciones y de las propias dependencias estatales.

\section{Referencias bibliográficas}

Cosciuc, Lucía Pilar (2018). Eran insostenibles todos los roles. Reflexiones y aportes para (re)conocer las vivencias de mujeres-madres-estudiantes universitarias (Tesina de Grado). Universidad Nacional de Rosario, Argentina.

Escobar, Manuel (2007). Universidad, conocimiento y subjetividad. Relaciones de saber/poder en la academia contemporánea. Revista Nómadas, (27), 48-61. http://www.redalyc.org/ pdf/1051/105116595005.pdf

Esquivel, Valeria (2011). La Economía del Cuidado en América Latina: poniendo a los cuidados en el centro de la Agenda. Panamá, PNUD.

Fabbri, Luciano (2014). Ni meramente natural, ni remotamente universal: Avatares de la teoría sexo/género. Revista Izquierdas, (19).

Federici, Silvia (2013). Revolución en punto cero. Madrid, España, Traficantes de sueños.

Fontanela, Marta (2008). “¿Qué es el patriarcado?”. En S. Gamba (Ed.) Diccionario de estudios de género y feminismos. Buenos Aires, Argentina, Biblos. Recuperado de: http://www.mujeresenred.net/IMG/article PDF/article a1396.pdf 
La "triple combinación de roles": mujeres-madres-estudiantes universitarias.

Aportes para (re)conocer y recuperar sus vivencias.

Lucía Cosciuc

Guzmán Gómez, Carlota (2011). Avances y retos en el conocimiento sobre los estudiantes mexicanos de educación superior en la primera década del siglo XXI. Revista Perfiles educativos, 33, 91-101. http://www.scielo.org.mx/pdf/peredu/v33nspe/v33nspea9.pdf

Haraway, Donna (1995). Ciencia, cyborgs y mujeres. La reinvención de la naturaleza. Madrid, España, Cátedra.

Harding, Sandra (1993). "Rethinking Standpoint Epistemology: 'What Is Strong Objectivity?”. En L. Alcoff, y E. Potter (Eds.), Feminist Epistemologies. Nueva York, Estados Unidos, Routledge.

Harding, Sandra (1998). Whose Science? Whose Knowledge? Thinking from women's lives. Nueva York, Estados Unidos, Cornell University.

Horton, Paul y Hunt, Chester (1986). Sociología. México, McGraw-Hill.

Palermo, Alicia (2006). El acceso de las mujeres a la educación universitaria. Revista argentina de Sociología, 4(7), 11-46. http://www.scielo.org.ar/scielo.php?script=sci arttext\&pi$\mathrm{d}=\mathrm{S} 166932482006000200002 \& \operatorname{lng}=\mathrm{es} \& \ln \mathrm{ln}=\mathrm{es}$

Palomar Verea, Cristina (2009). Maternidad y mundo académico. Alteridades, 19(38), 55-73. http://www.scielo.org.mx/pdf/alte/v19n38/v19n38a5.pdf

Rodríguez Enríquez, Corina (2001). "Economía del cuidado, equidad de género y nuevo orden económico internacional". En A. Giron y E. Correa (Eds.), Del Sur hacia el Norte: Economía politica del orden económico internacional emergente. Buenos Aires, Argentina, CLACSO.

Saletti Cuesta, Lorena (2008). Propuestas teóricas feministas en relación al concepto de maternidad. Revista Clepsydra, 7, 169-183.

Samudio, Edda (2016). El acceso de las mujeres a la educación superior. La presencia femenina en la Universidad de Los Andes. Revista Procesos Históricos, (29), 77-101. http://www. redalyc.org/pdf/200/20044209005.pdf

Vaggione, Juan Marco (2008). "Las familias más allá de la heteronormatividad”. En C. Motta y M. Sáez (Eds.), La mirada de los jueces. Sexualidades diversas en la jurisprudencia Latinoamericana. Bogotá, Colombia, Siglo del Hombre Editores. Recuperado de: https://programaddssrr.files. wordpress.com/2013/05/la-mirada-de-los-jueces-las-familias-mc3a1s-allc3a1-de-la-heteronormatividad.pdf

Veroneze, Renato (2015). Ágnes Heller, cotidianidad e individualidad: fundamentos para la conciencia ética y política del ser social. Revista Trabajo Social, (17), 131-144.

Recibido: 23/04/2020

Aceptado: $18 / 11 / 2020$ 